Faculty of Mathematical Sciences

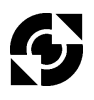

University of Twente

The Netherlands
P.O. Box 217

7500 AE Enschede

The Netherlands

Phone: +31-53-4893400

Fax: +31-53-4893114

Email: memo@math.utwente.nl

www.math.utwente. $\mathrm{nl} /$ publications

Memorandum No. 1672

The standard set game

of a cooperative game

A.F. Bumb and C. Hoede

February, 2003

ISSN 0169-2690 


\title{
The standard set game of a cooperative game
}

\author{
A. Bumb and C. Hoede \\ Department of Applied Mathematics \\ University of Twente \\ 7500 AE Enschede \\ The Netherlands
}

\begin{abstract}
We show that for every cooperative game a corresponding set game can be defined, called the standard set game.

Values for set games can be applied to this standard game and determine allocations for the cooperative game. On the other hand, notions for cooperative games, like the Shapley value, the $\tau$-value or the core can be considered in the context of the standard set games.
\end{abstract}

Key words: cooperative game, set game

AMS classification: $91 \mathrm{~A} 44$

\section{Introduction}

The concept set game was introduced by Hoede in [H 92]. A set game is a pair $\left\langle N_{s}, v_{s}\right\rangle$, where $N_{s}$ is a nonempty, finite set, called the player set and $v_{s}: 2^{N_{s}} \rightarrow 2^{U_{s}}$ associates with every coalition $S$ of players a subset of $U_{s}$, called the value (worth) of coalition $S$. We assume that $v_{s}(\emptyset)=\emptyset$. Set games differ from cooperative games in the fact that the value of a coalition $S$ of $N_{s}$ players is not a real number, but a set taken from a universe $U_{s}$.

Example 1 Let $U_{s}=\{a, b, c\}$ be the universe and $N_{s}=\{1,2,3\}$ be the set of players. The coalitions have the values

$$
\begin{aligned}
v_{s}(\emptyset) & =\emptyset \\
v_{s}(\{1\}) & =\{a\} \\
v_{s}(\{2\}) & =\{a, b\} \\
v_{s}(\{3\}) & =\{a, b, c\} \\
v_{s}(\{1,2\}) & =\{a, b\} \\
v_{s}(\{1,3\}) & =\{a, b, c\} \\
v_{s}(\{2,3\}) & =\{a, b, c\} \\
v_{s}\left(N_{s}\right) & =\{a, b, c\} .
\end{aligned}
$$

A solution $\psi$ on the set of all set games $G$ associates a so-called allocation $\psi\left(N_{s}, v_{s}\right)=$ $\left(\psi_{i}\left(N_{s}, v_{s}\right)\right)_{i \in N_{s}} \in\left(2^{U_{s}}\right)^{N_{s}}$ with every set game $\left\langle N_{s}, v_{s}\right\rangle$. For every $i \in N_{s}, \psi_{i}$ represents 
the items that are given, according to the solution $\psi$, to player $i$ from participating in the game.

Several solutions were proposed for set games (see [A94], [DS01a], [DS01b] and [SZL99]). As stated in [DS01b], these solutions can be included in the class of semi-marginalistic values. A semi-marginalistic value $\psi$ on the set game space $G$ has the following form

$$
\psi_{i}\left(N_{s}, v_{s}\right)=\bigcup_{\substack{S \subseteq N_{s} \\ S \ni i}}\left[v_{s}(S)-\nabla_{S, i}^{v_{s}}\right] \text {, for all }\left\langle N_{s}, v_{s}\right\rangle \in G \text { and all } i \in N_{s},
$$

where $\nabla_{S, i}^{v_{s}}$ is a set determined by the worth of a certain collection of coalitions, somehow determined by $S$ and/or $i$. For example, by choosing $\nabla_{S, i}^{v_{s}}:=v_{s}(S-\{i\})$ one obtains the individually marginalistic value (IM) introduced in [A94], by choosing $\nabla_{S, i}^{v_{s}}:=\bigcup_{j \in S} v_{S}(S-$ $\{j\}$ ) one obtains the the overall-individually marginalistic value (OIM) introduced in [A94], by choosing $\nabla_{S, i}^{v_{S}}:=\bigcup_{T \subset S} v_{s}(T)$ one obtains the overall-conditionally marginalistic value (OCM) introduced in [SZL99], and, finally, by choosing $\nabla_{S, i}^{v_{s}}:=\bigcup_{T \subseteq N_{s}-i} v_{s}(T)$ one obtains the Driessen-Sun value (DS) introduced in [DS01a].

Example 2 The $I M$ solution to the set game introduced in Example 1 is the following

$$
\begin{aligned}
& \psi_{1}=\{a\} \\
& \psi_{2}=\{a, b\} \\
& \psi_{3}=\{a, b, c\} .
\end{aligned}
$$

In many situations, there is a cost associated to each element of the universe $U_{s}$ and one is interested in how to share the costs between the players. One method of cost sharing is proposed by Hoede in [H02]. The allocation of the set game determines for each player $i, \psi_{i}$ as a set of elements of $U_{s}$. If an element $u_{S}, S \subseteq N$ has cost $c\left(u_{S}\right)$, then this cost is to be shared by all players that have $u_{S}$ in their allocation. In the literature people consider cooperative games on the reals, in the sense that the worth of a coalition is a real number, usually chosen from $\mathbb{R}$. Our goal is to show the intimate relationship between cooperative games and set games.

This paper is structured as follows. In Section 2 we show that with every cooperative game $\left(N_{c}, v_{c}\right)$ one can associate a set game $\left\langle N_{s}, v_{s}\right\rangle$, using basic units. In Sections 3,4 and 5 we will analyze the relations between several solutions defined for a cooperative game and the solutions defined on the associated standard set game.

\section{The standard set game}

Consider a cooperative game $\left(N_{c}, v_{c}\right)$, where $N_{c}$ is the set of players and $v_{c}: 2^{N_{c}} \mapsto \mathbb{R}$ is a mapping which associates with each coalition $S$ the value of the coalition. We associate with $\left(N_{c}, v_{c}\right)$ the following set game, called the standard set game associated to $\left(N_{c}, v_{c}\right)$.

The universe $U_{s}$ is $U_{s}=\left\{u_{S^{\prime}} \mid S^{\prime} \subset N_{c}\right\}$, where the elements $u_{S}$ are called basic units. The set of players $N_{s}$ is $N_{s}=N_{c}$ and $v_{s}: 2^{N_{s}} \rightarrow 2^{U_{s}}$ is defined by

$$
v_{s}(\emptyset)=\emptyset
$$


and

$$
v_{s}(S)=\left\{u_{S^{\prime}} \mid S^{\prime} \subset S, S^{\prime} \neq \emptyset\right\} .
$$

Every basic unit $u_{S}, S \subset N_{s}$, has a cost

$$
c\left(u_{S}\right)=\sum_{T \subseteq S}(-1)^{|S|-|T|} v_{c}(T) .
$$

One can easily verify that for each $S, S \subset N_{s}$,

$$
v_{c}(S)=\sum_{T \subseteq S} c\left(u_{T}\right) .
$$

Remark Note that (2) implies that if for every $S \subseteq N, c\left(u_{S}\right) \geq 0$, then the cooperative game $\left(N_{c}, v_{c}\right)$ is monotonic, i.e., if $S \subseteq T$ than $v(S) \leq v(T)$, for every $S \subseteq N$ and $T \subseteq N$.

\section{Example 3}

Consider the following cooperative game. $N_{c}=\{1,2,3\}$ and

$$
\begin{aligned}
v_{c}(\emptyset) & =0 \\
v_{c}(1) & =1 \\
v_{c}(2) & =2 \\
v_{c}(3) & =3 \\
v_{c}(1,2) & =41 \\
v_{c}(1,3) & =42 \\
v_{c}(2,3) & =43 \\
v_{c}\left(N_{c}\right) & =101 .
\end{aligned}
$$

The standard set game associated with $\left(N_{c}, v_{c}\right)$ is defined by $U_{S}=\left\{u_{S^{\prime}} \mid S^{\prime} \subset N_{c}\right\}, N_{s}=N_{c}$ and

$$
\begin{aligned}
v_{s}(\emptyset) & =\emptyset \\
v_{s}(\{1\}) & =\left\{u_{\{1\}}\right\} \\
v_{s}(\{2\}) & =\left\{u_{\{2\}}\right\} \\
v_{s}(\{3\}) & =\left\{u_{\{3\}}\right\} \\
v_{s}(\{1,2\}) & =\left\{u_{\{1\}}, u_{\{2\}}, u_{\{1,2\}}\right\} \\
v_{s}(\{1,3\}) & =\left\{u_{\{1\}}, u_{\{3\}}, u_{\{1,3\}}\right\} \\
v_{s}(\{2,3\}) & =\left\{u_{\{2\}}, u_{\{3\}}, u_{\{2,3\}}\right\} \\
v_{s}\left(N_{s}\right) & =\left\{u_{\{1\}}, u_{\{2\}}, u_{\{3\}}, u_{\{1,2\}}, u_{\{1,3\}}, u_{\{2,3\}}, u_{\{1,2,3\}}\right\}
\end{aligned}
$$


From (1), the costs of the basic units are

$$
\begin{aligned}
c\left(u_{\{1\}}\right) & =1 \\
c\left(u_{\{2\}}\right) & =2 \\
c\left(u_{\{3\}}\right) & =3 \\
c\left(u_{\{1,2\}}\right) & =38 \\
c\left(u_{\{1,3\}}\right) & =38 \\
c\left(u_{\{2,3\}}\right) & =38 \\
c\left(u_{\{1,2,3\}}\right) & =-19 .
\end{aligned}
$$

\section{Remark}

In [A94] and [DS01b] it is proved that for every monotonic set game, i.e., a game for which $v_{s}(T) \subseteq v_{s}(S)$, for every $T \subseteq S \subseteq N_{s}$, the $I M$ and $O I M$ values coincide.

Clearly, the standard set game $\left\langle N_{s}, v_{s}\right\rangle$ associated with a cooperative game $\left(N_{c}, v_{c}\right)$ is always monotonic. Hence, $\operatorname{IM}\left(N_{s}, v_{s}\right)=\operatorname{OIM}\left(N_{s}, v_{s}\right)$.

\section{The Shapley value}

In this section we will first show two ways of obtaining the Shapley value for a cooperative game $\left(N_{c}, v_{c}\right)$ via the standard set game associated with it.

Let $\left(N, v_{c}\right)$ be a cooperative game and $\left\langle N, v_{s}\right\rangle$ its associated standard set game. Recall that the Shapley value for $\left(N, v_{c}\right)$ is defined as

$$
S h_{i}\left(N, v_{c}\right)=\sum_{\substack{S \subseteq N \\ S \ni i}} \frac{(|S|-1) !(|N|-|S|) !}{|N| !}[v(S)-v(S-\{i\})] .
$$

An equivalent definition of the Shapley value is

$$
S h_{i}\left(N, v_{c}\right)=\sum_{\substack{S \subseteq N \\ S \ni i}} \frac{1}{|S|} \sum_{T: T \subset S}(-1)^{|S|-|T|} v_{c}(T) .
$$

Now consider the solutions $I M, O C M$ and $D S$ for $\left\langle N, v_{s}\right\rangle$. From the definition of these solutions (see Section 1) and the definition of a standard set game follows that

$$
\psi_{i}=I M\left(N, v_{s}\right)=O C M\left(N, v_{s}\right)=D S\left(N, v_{s}\right)=\bigcup_{S \ni i}\left\{u_{S}\right\}
$$

Consider for this $\psi_{i}$ the cost sharing method $a=\left(a_{i}\right)_{i \in N}$ defined as described before, leading to

$$
a_{i}=\sum_{\substack{S \subseteq N \\ S \ni i}} \frac{1}{|S|} c\left(u_{S}\right)
$$

From (1) follows that

$$
S h_{i}\left(N, v_{c}\right)=a_{i}
$$


Next we will show how, for a restricted class of games, the Shapley value comes forward with the help of the excess vector (complaint vector). Suppose that $I\left(v_{c}\right) \neq \emptyset$. For every $x \in I\left(v_{c}\right)$, the excess vector (complaint vector) $\theta(x)$ has as its coordinates the excesses

$$
e(S, x):=v_{c}(S)-\sum_{i \in S} x_{i}, \text { for every coalition } S,
$$

and these excesses are written down in decreasing order. The imputation for which the complaint vector is lexicographically minimal is called the nucleolus.

Using (2) the excesses can be written as

$$
e(S, x)=\sum_{T \subseteq S} c\left(u_{T}\right)-\sum_{i \in S} x_{i} .
$$

Suppose now that $c\left(u_{S}\right) \geq 0$, for all $S \subseteq N$, and that we are interested in finding an imputation $x$ that keeps all the excesses negative. A natural way is to proceed as follows.

First set $x_{i}=c\left(u_{i}\right)$ for each $i \in N_{s}$. Clearly, $e(\{i\}, x) \leq 0$, for every $i \in N_{s}$ but for $S$ with $|S| \geq 2$ it may happen that $e(S, x)>0$. Next we change $x$ such that

$$
e(S, x) \leq 0 \text { for every } S \subseteq N,|S| \leq 2 .
$$

Clearly,

$$
x_{i}=c\left(u_{\{i\}}\right)+\frac{1}{2} \sum_{\substack{T \subseteq S \\|T|=2}} c\left(u_{T}\right), \text { for every } i \in N,
$$

satisfies (5). Proceeding in this way, we finally obtain that the imputation

$$
x_{i}=\sum_{\substack{S \subseteq N \\ S \ni i}} \frac{1}{|S|} c\left(u_{S}\right)
$$

maintains all the excesses non-positive. Obviously, $x$ is exactly the Shapley value for $\left(N, v_{c}\right)$.

We have seen here how one can use the standard set game in order to obtain the Shapley value. It is very difficult to extend the approach in order to obtain the nucleolus. The reason for this is that the analytic minimization involved in calculating the nucleolus is unavoidable and beyond the scope of set games. A similar phenomenon occurs in the next section.

\section{The $\tau$ - value}

As before, let $\left(N, v_{c}\right)$ be a cooperative game with $n$ players. The $\tau$-value is based on two vectors $M\left(v_{c}\right)$ and $m\left(v_{c}\right)$. The vector $M\left(v_{c}\right)$, respectively $m\left(v_{c}\right)$, has as $i$-th coordinate

$$
M_{i}\left(v_{c}\right):=v(N)-v(N-\{i\}),
$$

respectively,

$$
m_{i}\left(v_{c}\right):=\max _{S: i \in S}\left(v_{c}(S)-\sum_{j \in S-\{i\}} M_{j}(v)\right) .
$$


The $\tau$-value $\tau_{c}$ of the game $\left(N, v_{c}\right)$ is the unique convex combination, when such a combination exists, of $m\left(v_{c}\right)$ and $M\left(v_{c}\right)$ lying in the hyperplane $E=\left\{x \in \mathbb{R}^{n} \mid \sum_{i=1}^{n} x_{i}=\right.$ $\left.v_{c}(N)\right\}$. Actually, the $\tau$-value is defined for games for which which this convex combination exists i.e., for quasi-balanced games.

Following the idea behind the $\tau$-value for cooperative games, for standard set games we may proceed as follows. For $M_{i}$ we choose what a player can get from the coalitions to which he belongs and for $m_{i}$ what he can get by himself. This leads to the choices

$$
M_{i}\left(v_{s}\right)=\sum_{S: i \in S} c\left(u_{S}\right)
$$

and

$$
m_{i}\left(v_{s}\right)=c\left(u_{i}\right) .
$$

Note that the vector $M\left(v_{c}\right)$ is in fact equal to $M\left(v_{s}\right)$.

Again, we are interested to find a convex combination between $m\left(v_{s}\right)$ and $M\left(v_{s}\right)$, if it exists, that lies in the hyperplane $E$ defined above. In other words, we are interested in finding an $\alpha \in[0,1]$ such that

$$
\sum_{i \in N}\left[\alpha m_{i}\left(v_{s}\right)+(1-\alpha) M_{i}\left(v_{s}\right)\right]=v_{c}(N)
$$

It is easy to see that such an $\alpha$ exists only if the set game satisfies the following two conditions:

$$
m_{i}\left(v_{s}\right) \leq M_{i}\left(v_{s}\right) \text {, for every } i \in N
$$

and

$$
\sum_{i \in N} m_{i}\left(v_{s}\right) \leq v_{c}(N) \leq \sum_{i \in N} M_{i}(v s)
$$

Then, $\alpha$ would be equal to

$$
\alpha=\frac{\sum_{i \in N} M_{i}\left(v_{s}\right)-v_{c}(N)}{\sum_{i \in N} M_{i}\left(v_{s}\right)-\sum_{i \in N} m_{i}\left(v_{s}\right)}
$$

or, in terms of basic units,

$$
\alpha=\frac{\sum_{i \in N} \sum_{S \ni i} c\left(u_{S}\right)-\sum_{S \subseteq N} c\left(u_{S}\right)}{\sum_{i \in N} \sum_{S \ni i} c\left(u_{S}\right)-\sum_{i \in N} c\left(u_{i}\right)} .
$$

Note that if it exists, the allocation given by the convex combination in (6) is not necessarily equal to the $\tau$-value associated to the cooperative game. The reason for this is that analytical expressions such as maximum and minimum of a function cannot be covered by a set game.

Remark Applying this procedure to the set game in Example 3 we obtain $\alpha=\frac{4}{9}$ and the following solution for $\tau_{s}=\left(\tau_{s, 1}, \tau_{s, 2}, \tau_{s, 3}\right)$ :

$$
\tau_{s, 1}=32 \frac{2}{3}, \quad \tau_{s, 2}=33 \frac{2}{3}, \quad \tau_{s, 3}=34 \frac{2}{3} .
$$




\section{The standard set game and the core}

For a cooperative game $\left(N_{c}, v_{c}\right)$ define the set of imputations $I\left(v_{c}\right)$ by

$I\left(v_{c}\right)=\left\{x \in \mathbb{R}^{|N|} \mid x_{i} \geq v_{c}(i)\right.$, for every $\left.i \in N\right\}$. The core of the game $\left(N_{c}, v_{c}\right)$ is the set of imputations satisfying $x(S) \geq v(S)$ for every $S \subseteq N$ and $x(N)=v(N)$, where for a set $S, x(S)=\sum_{i \in S} x_{i}$. Equivalently, the core is the set of imputations that keep all the excesses non-positive.

Using (2) we can rewrite the definition of the core as the set of all imputations $x$ satisfying $x(S) \geq \sum_{T \subseteq S} c\left(u_{T}\right)$ and $x(N)=\sum_{T \subseteq N} c\left(u_{T}\right)$.

First of all remark that if, in the standard set game, $c\left(u_{S}\right) \geq 0$ for every $S \subseteq N$, then the core is nonempty. In Section 3 we already saw that in this case the Shapley value has the property that it makes the excesses non-positive. So it is in the core.

In fact, the nonnegativity of the costs of the basic units implies a stronger statement with respect to $\left(N_{c}, v_{c}\right)$, namely that the game is convex. Recall that $\left(N_{c}, v_{c}\right)$ is convex if

$$
v(S)+v(T) \leq v(S \cap T)+v(S \cup T) \text {, for every } S \subseteq N, T \subseteq N .
$$

Using (2), the convexity condition above can be rewritten as

$$
\sum_{\substack{S^{\prime} \subseteq S \cup T \\ S^{\prime} \cap(S T) \neq \emptyset \\ S^{\prime} \cap(T \backslash S) \neq \emptyset}} c\left(u_{S^{\prime}}\right) \geq 0 \text {, for every } S \subseteq N, T \subseteq N .
$$

It follows immediately that a game for which all basic units have nonnegative costs is convex. Hence, the core is nonempty and the Shapley value is an element of it.

One would like to formulate a necessary and sufficient condition for the non-emptiness of the core in terms of the costs of the standard set game elements. One way to achieve this is by simply translating the characterization of non-emptiness given by Bondareva [B63] and Shapley [S67]. The conditions are that for each set of values $\alpha_{S}, S \subseteq N$, for which we have that

$$
\sum_{S \ni i} \alpha_{S}=1
$$

it holds that

$$
\sum_{S \subseteq N} c\left(u_{S}\right)\left(1-\sum_{S \subseteq S^{\prime}} \alpha_{S^{\prime}}\right) \geq 0 .
$$

Again, we immediately see that this condition is satisfied if $c\left(u_{S}\right) \geq 0$, for all $S \subseteq N$. However, we would like to formulate necessary and sufficient conditions on the $c\left(u_{S}\right)$ only. So far we have not succeeded in this.

\section{Discussion}

The axiomatization of values for set games was first studied by Aarts, Funaki and Hoede ([AFH97] and [AFH00]). The papers of Driessen and Sun ([DS01a] and [DS01b]) form a continuation of this work.

In first instance it was somewhat unclear how set games compare to cooperative games. By the results given in this paper we now know that set games are intimately related to cooperative games with coalition values in the reals. However, they cover only the 
combinatorial aspect of normal cooperative games. It was for this reason that direct analogs for the $\tau$-value or the nucleolus could not be found for the standard set game.

Yet the fact that set games may be seen as covering the combinatorial aspect of cooperative games has a certain potential. As was shown in [H02], Example 1 is the set game that can be defined for the cost sharing problem for three pieces $a, b$ and $c$, of a landing strip, to be used by players, planes 1,2 and 3, where 1 uses $a, 2$ uses $a$ and $b$ and 3 uses $a, b$ and $c$. The solution given in Example 2 gives the basis for the cost sharing solution by remarking that all the players should pay for $a$, players 2 and 3 for $b$ and player 3 for $c$. The actual costs of the basic units $a, b$ and $c$ are left out of consideration. This situation seems to prevail in many cost sharing problems. The players are interested in certain basic units and coalitions can be given a worth equal to the union of these basic units. The set game that is defined this way captures the combinatorial aspects of the cost sharing problem. Further considerations on the actual costs of the basic units may then include the cost sharing based on the set game solution as one of the inputs. This splitting up of cost sharing problems into a set game part and a part in which only costs are further considered will be discussed in another paper.

\section{References}

[A94] H. Aarts, Minimum cost spanning tree games and set games, Ph.D. thesis, Faculty of Mathematical Sciences, University of Twente, Enschede, The Netherlands, 1994.

[AFH97] H. Aarts, Y. Funaki and C. Hoede, A marginalistic value for monotonic set games, International Journal of Game Theory 26, pages 97-111, 1997.

[AFH00] H. Aarts, Y. Funaki and C. Hoede, Set games, Homo Oeconomicus XVII (1/2), pages 137-154, ACCEDO Verlagsgesellschaft, Munchen, 2000.

[B63] O. N. Bondareva, Some applications of linear programming to cooperative games. Problemy Kibernetiki, 10, pages 119-139, 1963.

[DS01a] T. S. H. Driessen, H. Sun, A potential approach to solutions for set games, Memorandum 1571, Faculty of Mathematical Sciences, University of Twente, Enschede, The Netherlands, 2001.

[DS01b] T. S. H. Driessen, H. Sun, A uniform approach to semi-marginalistic values for set games, Memorandum 1572, Faculty of Mathematical Sciences, University of Twente, Enschede, The Netherlands, 2001.

[H92] C. Hoede, Graphs and games, Memorandum 1065, Faculty of Mathematical Sciences, University of Twente, Enschede, The Netherlands, 1992.

[H02] C. Hoede, A note on set games and cost sharing problems, Memorandum 1642, Faculty of Mathematical Sciences, University of Twente, Enschede, The Netherlands, 2002.

[S67] L.S. Shapley, On balanced sets and cores, Naval Research Logistics Quarterly 14, pages 453-460, 1967. 
[SZL99] H. Sun, S. Zhang and X. Li, A coalitional power value for set games. Working paper, Department of Applied Mathematics, Northwestern Polytechnical University, Xi'an, Shaanxi, China, 1999. 